Archived version from NCDOCKS Institutional Repository http://libres.uncg.edu/ir/asu/

\title{
Appalachľan
}

$\overline{\text { B O O N E, N O R T H C A R O L I N A }}$

\section{The Association Between Oral Microorgansims And Aspiration Pneumonia In The Institutionalized Elderly: Review And Recommendations}

\author{
Authors \\ Cherin C. Pace \& Gary H. McCullough
}

\begin{abstract}
Aspiration pneumonia is a leading cause of illness and death in persons who reside in long-term-care facilities and, combined with the lack of proper oral health care and services, the risk of aspiration pneumonia rises. The purpose of this article is to review recent literature on oral hygiene and oral care in long-term-care facilities and report new findings regarding associated risks for aspiration pneumonia, as well as research on oral care and health outcomes. The PubMed MeSH database was utilized to direct a specific search by entering terms "aspiration pneumonia" and "oral hygiene" from 1970 to 2009, which yielded 34 articles. The Ovid and Google Scholar databases were utilized as well and provided no additional references for the two terms. A manual search of references from other articles, including three systematic reviews published over the past decade, provided additional information regarding oral microorganisms and respiratory pathogens, as well as investigations of oral care. Finally, a brief but comprehensive introductory review was organized regarding oral microorganisms, biofilm, periodontal disease, and pneumonia to establish a framework for discussion. Over- all, studies suggest (1) an association between poor oral hygiene and respiratory pathogens, (2) a decrease in the incidence of respiratory complications when patients are provided chemical or mechanical interventions for improved oral care, (3) the complex nature of periodontal disease and aspiration pneumonia make direct connections between the two challenging, and (4) additional studies are warranted to determine adequate oral hygiene protocols for nursing home patients to further reduce the incidence of aspiration pneumonia.
\end{abstract}




\title{
The Association Between Oral Microorgansims and Aspiration Pneumonia in the Institutionalized Elderly: Review and Recommendations
}

\author{
Cherin C. Pace · Gary H. McCullough
}

Aspiration pneumonia occurs when regurgitated gastric contents or oropharyngeal secretions are inadvertently directed into the trachea and subsequently into the lungs. As the bacteria and other microorganisms become part of an infiltrate within the lung tissue, the resulting effect is an infection in the lung, either bilaterally or unilaterally. Research shows that the right lower lobe is the most frequent site of infiltrates (visualized via chest radiography). After urinary tract infections, aspiration pneumonia is the most common infection in nursing home residents, the most common reason for transfer to the hospital, and the leading cause of death from infection [1]. Nursing home residents, particularly those with a history of neurologic disease, are at risk for dysphagia and, ultimately, aspiration pneumonia [1]. Residents of long-term care facilities are prone to poor oral health due to lack of oral hygiene care as well as conditions of periodontal and/or dental disease. Some research suggests that the organisms present in the oropharyngeal secretions of an individual with oral disease can be particularly dangerous if aspirated into the lungs of a medically compromised patient.

The purpose of this article is to review recent literature on oral hygiene and oral care in long-term-care facilities and report new findings on associated risks for aspiration 
pneumonia and research on oral care and health outcomes. While the association between dysphagia and aspiration pneumonia has been reported in the literature and is certainly pertinent to this topic, this review focuses on the role of oral microorganisms and respiratory compromise which may occur in conjunction with a deglutition disorder. The PubMed MeSH database was utilized to direct a specific search by entering the terms "aspiration pneumonia" and "oral hygiene" from 1970 to 2009, which yielded 34 articles. The Ovid and Google Scholar databases were utilized as well and provided no additional references for the two terms. A manual search of references from other articles, including three systematic reviews published over the past decade, provided additional information regarding oral microorganisms and respiratory pathogens, as well as investigations of oral care.

A brief but comprehensive introductory review of oral microorganisms, biofilm, periodontal disease, and pneumonia is provided to establish a framework for discussion.

\section{Normal Oral Flora and Biofilm}

To provide a comprehensive look at the association between aspiration pneumonia and oral microorganisms, one must have an understanding of the microorganisms present in the oral cavity in health as well as in disease. In utero, the oral cavity is sterile, but shortly after birth, within a few hours to one day, a simple oral flora develops. Microorganisms are transmitted to the infant from its mother and other family members and caretakers. As the infant grows, introduction of microorganisms is ongoing and complex. Many of the salivary bacteria come from the dorsum of the tongue, but some are from mucous membranes as well as gingival and periodontal tissues. High counts of microorganisms are found in dental biofilm, periodontal pockets, and carious lesions [2].

First described in the mid-1670s by Dutch scientist Anton van Leeuwenhoek, bacteria are the simplest organisms and can be seen only by microscope. There are thousands of species of bacteria, most of which are not harmful to humans (innocuous). Species of bacteria that are harmful to humans are called pathogenic or virulent and are capable of causing disease. Both innocuous and pathogenic bacteria live in symbiotic relationship within the oral cavity. Bacteria can replicate quickly, which enables them to adapt rapidly to changes in their environment. The identification and classification of bacteria is based largely upon the composition of the cell wall. When stained with crystal violet dye, bacteria with a thick, single-cell wall will retain a purple color and are identified as Gram positive. Gram-negative bacteria have double-cell walls and do not retain the purple stain. These Gram-negative bacteria play an important role in the tissue destruction seen in periodontal (gum) disease [3].

Bacteria can be categorized into three groups based on their need for oxygen: (1) aerobic bacteria require oxygen to live, (2) anaerobic bacteria cannot live in the presence of oxygen, and (3) facultative anaerobic bacteria can live with or without oxygen. In addition, bacteria can be free-floating (planktonic) or attached. It is estimated that $99 \%$ of bacteria on earth live as attached bacteria. Once bacteria become attached to a surface, a different set of genes are activated than when free floating; this different set of genes gives the bacteria different characteristics [3].

Attached bacteria can adhere to surfaces and to one another, forming a well-organized community of bacteria that are described as living in a biofilm. A biofilm can be formed by single bacterial species but usually consists of many species of bacteria as well as other organisms and debris and becomes embedded in an extracellular slime layer. Biofilms can form rapidly on most wet surfaces and there are many types, including plaque on teeth, slime in fish tanks, slime deposits that clog the sink drain, indwelling IV and urinary catheters, and prosthetic devices (heart valves, biliary stents, pacemakers, artificial joints). It was a biofilm within a hotel air conditioning system that was responsible for the 1976 outbreak of Legionnaires disease which killed 29 people [3].

As dental plaque matures, Gram-negative, anaerobic, periodontal pathogens colonize within biofilms and multiply. According to Socransky and Haffajee [4], biofilms are composed of microcolonies of bacterial cells randomly distributed within a "glycocalyx" (thick, slimy shell). Dental biofilms are potentially "the most complex biofilms that exist in nature" due to the nonshedding, coarse tooth surface where bacteria attach, the constant flow of nutrients in the oral cavity, and the coaggregation (relationships) of various species within the biofilm.

Infections caused by biofilms are persistent, chronic, and difficult to eradicate as the pathogenic species within the biofilms reproduce in large numbers and are widely distributed within the oral cavity. They exist in communities that work together to provide protection against host defense mechanisms and treatments and commonly attach to new surfaces of the host or to organisms already attached to the host. It is the spreading and recolonization that make biofilms a persistent threat [3, 4].

\section{Microorganisms in Oral Disease}

\section{Oral Bacteria}

The pathogenicity of oral biofilms depends on the number and types of oral bacteria, which vary considerably from 
health to mild gingivitis (gum inflammation) to periodontitis (advanced gum disease). In a healthy mouth, only a very small proportion of these are capable of causing periodontal disease. Bacteria associated with periodontal disease are a different type than those found in healthy mouths, with varying bacterial composition from patient to patient and from site to site within the same mouth. Chronic periodontitis is associated with high proportions of Gram-negative and motile bacteria. Table 1 represents these variations in terms of Gram stain, motility, and oxygen requirement.

While more than 500 species of bacteria have been isolated from one periodontal pocket, only a small percentage of these are considered periodontal pathogens. Table 2 provides a listing of the species of bacteria associated with periodontal disease [3].

In addition to bacteria, the oral microbiota includes Gram-positive and Gram-negative facultative and anaerobic cocci and rods, as well as spirochetes. The nature and numbers of organisms present in an individual's mouth vary depending on a number of factors, including the presence of other organisms, adhesion to those other organisms within plaque or biofilm, and adhesion from pits, fissures, and caries.

What a specific organism needs for nutrition can be provided by the host's diet, the host's specific tissues/ secretions, or other microorganisms (certain bacteria provide metabolites needed for other bacteria). Whether an individual has full dentures, is partially edentulous and wears partial dentures, or is dentate makes a difference in the types of microorganisms that may thrive. Salivary counts of lactobacilli and certain yeasts have been shown to be low in edentulous mouths but higher upon insertion of dentures, an indication that the dentures serve to mechanically retain the organism. These organisms are related to dental caries (tooth decay), with counts of more than 10,000 organisms per milliliter of saliva. Consider that if a person swallows $1000-1500 \mathrm{ml}$ of saliva per day, the level of lactobacilli ingested would be $10^{7}$ organisms. Spirochetes are not found in infants or edentulous adults (with or without dentures). They are highly motile; do not seem to adhere to other organisms, tooth, or tissue surfaces; and

Table 1 Bacteria associated with oral health and disease

\begin{tabular}{lll}
\hline Health & Gingivitis & Periodontitis \\
\hline Numbers found & Numbers found from & Numbers found from a \\
within a sulcus: & a specific site: & specific site: \\
$100-1000$ & $1000-100,000$ & $100,000-100,000,000$ \\
$75-85 \%$ are Gram & Equal proportion & Mostly Gram negative \\
positive & Gram positive and & \\
& Gram negative & \\
Most are & & \\
nonmotile & & \\
\hline
\end{tabular}

From [3] have specific growth requirements that can be met only via mechanical retention in the gingival crevice [5].

\section{Periodontal Disease}

Periodontal disease comprises a group of chronic inflammatory conditions that affect the supporting structures of the tooth (gums, periodontal ligament, and alveolar bone). As previously discussed, bacteria are responsible for the production of dental plaque which when first formed is soft and sticky. However, if undisturbed, plaque will mineralize and form hardened dental calculus. Together, the bacteria-laden plaque and calculus form in the subgingival sulcus resulting in a localized inflammatory process, gingivitis, the first stage of periodontal disease. As measured by periodontal probing, the gingival sulcus is the $1-3-\mathrm{mm}$ space between the tooth and gum in a healthy periodontium. If the inflammatory process continues, the junctional epithelium migrates apically and a periodontal pocket is formed. A periodontal pocket is the pathological deepening of the gingival sulcus, which measures $4 \mathrm{~mm}$ or greater [3]. At this point in the disease process (periodontitis), the alveolar bone and periodontal ligament fibers are destroyed. Gingivitis is an easily treatable, reversible form of periodontal disease. Conversely, periodontitis results in permanent damage to the supporting structures of the teeth, and if untreated it will result in tooth mobility and ultimately tooth loss.

A multibacterial etiology makes the diagnosis of active periodontal disease based on microbiological data difficult. Tanner et al. [6] reported that the microbiota of tongue samples was less sensitive than that of subgingival samples in detecting periodontal species, with overlap in species detected in health and early periodontitis. Haffajee et al. [7] compared the site prevalence of 40 subgingival species in 30 periodontally healthy persons (mean age $=36 \pm$ 9 years), 35 elders with a well-maintained periodontium (mean age $=77 \pm 5$ years), and 138 adult periodontitis subjects (mean age $=46 \pm 11$ years). Subgingival plaque samples were taken from the mesial aspect of each tooth (up to 28 samples) in the 203 subjects at baseline. The presence and levels of 40 subgingival taxa were determined in 5003 plaque samples using whole genomic DNA probes and checkerboard DNA-DNA hybridization. Clinical assessments, including duplicate measures of gingival redness, bleeding on probing, plaque accumulation, suppuration, pocket depth, and attachment level, were made at six sites per tooth. Four species were significantly elevated and at greater prevalence in the periodontitis group, suggesting an etiologic role for Bacteroides forsythus, Porphyromonas gingivalis, Treponema denticola, and Selenomonas noxia in adult periodontitis.

Socransky and Haffajee [4] discovered a direct association between large numbers of "red complex" bacteria, 
Table 2 Bacteria strongly associated with chronic periodontitis

\begin{tabular}{lll}
\hline Bacteria & Gram stain & Motility \\
\hline Actinobaccilus actinomycetem comians (serotype a) & Gram negative & Nonmotile \\
$\begin{array}{l}\text { Fusobacterium nucleatum subspecies nucleatum } \\
\text { Porphyromonas gingivalis (previously known } \\
\text { as Bacteroides gingivalis) }\end{array}$ & Gram negative & Nonmotile \\
Bacteroides forsythus (Tannerella forsythensis) & Gram negative & Nonmotile \\
Streptococcus intermedius & Gram negative & Nonmotile \\
Campylobacter rectus & Gram positive & Nonmotile \\
Eubacterium nodatum & Gram negative & Motile \\
Fusobacterium nucleatum subspecies polymorphum & Gram positive & Nonmotile \\
Prevotella intermedia & Gram negative & Nonmotile \\
Peptostreptococcus micros & Gram negative & Nonmotile \\
Prevotella nigrescens & Gram positive & Nonmotile \\
Treponema denticola & Gram negative & Nonmotile \\
\hline
\end{tabular}

commonly found in plaque when a periodontal infection occurs, and increased pocket depth and bleeding on probing, two clinical parameters important to the diagnosis of periodontal infection. Red complex bacteria include Porphyromonas gingivalis, Treponema denticola, and Tannerella forsynthensis. Other bacterial pathogens appear to be associated with the status and progression of periodontal disease. Up to one billion bacteria may be present in the pocket, and pockets range from 4 to $12 \mathrm{~mm}$ in depth.

When the periodontium is disturbed (during brushing, chewing, or tooth cleaning), Gram-negative bacteria found in dental biofilms release a variety of biologically active, toxic products, such as bacterial endotoxins known as lipopolysaccharides (LPS), protein toxins, chemotactic (adhesive) peptides, and organic fatty acids. These destructive molecules diffuse into the gingival epithelium, initiating an inflammatory response that first manifests as acute gingivitis. Gingival swelling can be accompanied by ulceration, allowing bacteria to transfer into the bloodstream (bacteremia). Because gingivitis may not be identified and treated for some time, chronic inflammation may lead to chronic bacteremia. As the body tries to protect itself with antibodies, neutrophils are released into connective tissue and cause further inflammation [4].

In addition to the inflammatory response, a type of protein cell called cytokine is released. Cytokines are produced by the immune cells to act as mediators, transmitting information or signals from one cell to another in order to influence the behavior of other cells [3]. These molecules, interleukin- $1 b$, interleukin-6, interleukin- 8 , and tumor necrosis factor- $a$, produce additional destructive molecules, including prostaglandin B2, which is responsible for the resorption of alveolar bone. Cytokines also activate destructive enzymes which break down epithelial cells, gingival tissue, and fibers attached to the root surface of the tooth. Once cytokines are activated, shallow gingival pockets deepen, the formation of periodontal pockets begins, and the loss of clinical attachment occurs [4].

Current data suggest that pathogens are necessary but not sufficient for disease activity to occur. In order for disease to arise from a pathogen, (1) it must be a virulent clonal type, (2) it must possess the chromosomal and extrachromosomal genetic factors to initiate disease, (3) the host must be susceptible to this pathogen, (4) the pathogen must be in numbers sufficient to exceed the threshold for that host, (5) it must be located at the right place, (6) other bacterial species must foster, or at least not inhibit, the process, and (7) the local environment must be one which is conducive to the expression of the species' virulence properties [4].

Dietary factors also affect the potential for periodontal disease by changing the quantity and the microbial composition of plaque [5]. Streptococcus mutans is dependent on the presence of dietary sucrose more so than glucose and will decrease in the presence of a carbohydrate-free diet, while the percentage of Streptococcus sanguis will increase. Increases in protein will result in a high percentage of Gram-positive, facultative, pleomorphic rods which are suspected to play a role in calculus formation. The consistency of the diet plays a role as well in that a soft diet will result in more gingivitis as opposed to a hard diet which is associated with more smooth surface plaque and caries.

Local factors contributing to disease include tooth morphology, calculus formation, damage from occlusal forces, food impaction, faulty dental restorations or appliances, and individual patient habits such as mouth breathing or inadequate self-care. Systemic factors for periodontal disease include stress, tobacco use, diabetes, osteoporosis, hormonal changes, inadequate nutrition, genetics, AIDS, and medications [3]. 
Pneumonia Defined and Classified

The definitions, descriptions, and categorizations of aspiration pneumonia in the literature are varied and perplexing, especially given the diversity of etiological factors.

Cavallazzi et al. [8] stated that aspiration pneumonitis and aspiration pneumonia are common entities that occur more frequently in populations that are susceptible to aspiration. In aspiration pneumonitis, the degree of lung injury caused by the aspiration of gastric contents is influenced by the $\mathrm{pH}$ and, to a lesser extent, the volume of the aspirate. In aspiration pneumonia, the key precipitating event is the inhalation of colonized oropharyngeal material.

Azarpazhooh and Leake [9] differentiate three subtypes of pneumonia: (1) Community-acquired Pneumonia (CAP) is prevalent with an incidence rate of 11.6 per 1,000 adults per year. CAP is responsible for approximately 500,000 hospitalizations in the United States, with outpatient costs of about $\$ 385$ million and inpatient costs of $\$ 8.4$ billion. The main causative agents for CAP are Streptococcus pneumoniae and Haemophilus influenzae; (2) Hospitalacquired Pneumonia (HAP), also known as nosocomial pneumonia, is a serious, life-threatening illness. According to the Centers for Disease Control (CDC), HAP accounts for $15 \%$ of all hospital-acquired infections, second only to urinary tract infections. The incidence rate of HAP is 22-44 per 10,000 patients over 60 years old. The mortality rate is especially high $(21-70 \%)$ for intensive care unit (ICU) patients. HAP generally occurs at least $48 \mathrm{~h}$ after hospital admission. Most susceptible are those who are mechanically ventilated. Ventilator-acquired pneumonia (VAP) has an incidence rate as high as $78 \%$ and remains high even with treatment. Each case of HAP/VAP can cost between $\$ 5800$ and $\$ 20,000$. The main causative agents of HAP are Staphylococcus aureus and Enterobacter; (3) Aspiration Pneumonia (AP) is reported in an estimated 200,000 cases per year, with over 15,000 deaths per year in the United States. About $45 \%$ of healthy adults aspirate while they sleep; however, a healthy person can clear secretions via forceful cough, while active ciliary transport and normal humoral and cellular immune mechanisms prevent the material from becoming infectious. AP can be caused by mechanical or cellular defense impairments; aspiration of large amounts of secretions, as seen in patients with neurologic or otherwise severe dysphagia; disruption of the gastroesophageal junction, anatomical abnormalities of the upper aerodigestive tract; or other types of swallow dysfunction [9].

Most cases of HAP can be attributed to aspiration of bacteria, which is also a primary cause of pneumonia in nursing home residents. It is this form of bacterial pneumonia, commonly occurring with aspiration and present in HAP and nursing home residents, to which this article primarily refers. Though not as common, contamination of the lower airways by microorganisms can also occur by inhalation of infectious aerosols, spread of infections from adjacent sites (intrapulmonary), or hematogenous spread from extrapulmonary sources of infection (Fig. 1).

\section{Association between Oral Hygiene and Pneumonia}

In 1998, Langmore and her colleagues [10] conducted a study to evaluate the contributions of specific risk factors to the development of aspiration pneumonia in elderly
Fig. 1 Pathogenesis of nosocomial bacterial pneumonia (from [9, p. 1466])

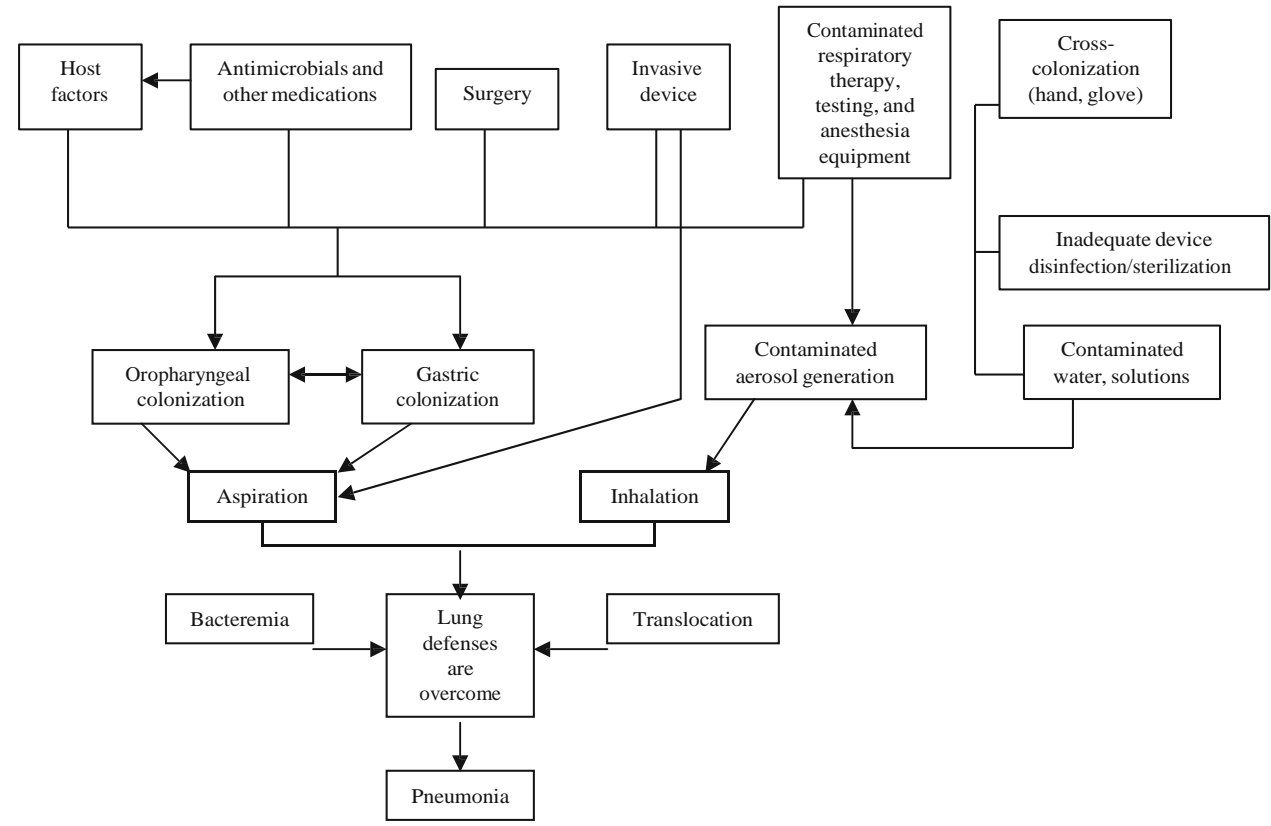


patients who were acutely ill and hospitalized, in nursing homes, or who were reasonably healthy outpatients. The risk factors evaluated included dysphagia, feeding status, functional status, medical status, and oral/dental status. The number of decayed teeth in individuals in all settings was associated with aspiration pneumonia ( $p W 0.01)$, as was occasional or no teeth brushing among dentate patients $(p W 0.01)$. Being edentulous had no effect. Other factors included dependence for oral care $(p W 0.01)$ and tube feeding ( $p=0.049$ ), which promotes colonization, in part, because of reduced salivary flow. The best predictors for pneumonia in dentate oral feeders were dependence for oral feeding ( $p W 0.01)$ and multiple medical diagnoses $(p=0.01)$.

In a 2002 follow-up [11], Langmore et al. focused on predictors of aspiration pneumonia in nursing home residents specifically. A total of 55 independent variables were examined to assess their association with the dependent variable, the presence of pneumonia. The risk factors that met the criterion of $p$ B 0.05 were retained. The prevalence of pneumonia within the study population was $3 \%$. Findings were similar to the prior study, but the prevalence of an increased number of medications $(50.7 \%)$, weight loss $(18.0 \%)$, urinary tract infections $(11.1 \%)$, and age over 85 years of age $(49.4 \%)$ were also reported as significant ( $p$ B 0.05 ). This group of predictors suggests that nursing home patients are more prone to chronic disease that slowly progresses and eventually leads to "decompensation" in functional status, nutritional status, pulmonary clearance, and immune status. As the immune system weakens, pneumonia becomes more and more difficult to resist.

Two systematic reviews $[9,12]$ have explored the relationship between oral hygiene and bacterial pneumonia in 2003 and 2006, respectively. Azarpazhooh and Leake [9] reported on four prospective cohort studies and one casecontrol study and found level II-2 evidence of a relationship between oral health and bacterial pneumonia. The presence of cariogenic and periodontal pathogens in dental plaque and saliva (odds ratio $[\mathrm{OR}]=4-9.6)$ and decayed teeth $(\mathrm{OR}$ is about 1.2 per decayed tooth) were identified as important risk factors. They also noted higher plaque scores were associated with a history of respiratory tract infection.

Scannapieco et al. [12] reported on 24 cohort studies. Two studies $[13,14]$ reported negative results regarding a relationship between oral hygiene and bacterial pneumonia. Both studies reported colonization of oral cavities in conjunction with pneumonia-related X-ray changes but stated results were not at all conclusive. Despite those two investigations, Scannapieco et al. [12] reported an overall relative risk of pneumonia at 9.6 when dental plaque was colonized, as well as a significant association between decayed teeth $(\mathrm{OR}=1.2)$, dental plaque $(\mathrm{OR}=4.2)$, and dependency for oral care $(\mathrm{OR}=2.8, p=0.03)$. Both systematic reviews noted that dentate patients in long-termcare settings were more likely to develop aspiration pneumonia than edentulous patients.

Two studies have provided valuable data since the two systematic reviews were completed. In 2008, 697 participants were evaluated for number of pneumonia-related deaths associated with periodontal disease as measured by the number of teeth with periodontal pockets (probing depths exceeding $4 \mathrm{~mm}$ ) [15]. Results showed that persons with 10 or more periodontal pockets had increased mortality rates from pneumonia compared with others. The incidence of periodontal pockets influences the prevalence of periodontal bacteria in the oral cavity. In elderly persons, aspiration pneumonia is caused almost entirely by anaerobic Gram-negative bacteria, such as periodontal bacteria. Anaerobic Gram-negative bacteria, including periodontal bacteria, produce high levels of methyl mercapitan $\left(\mathrm{CH}_{3} \mathrm{SH}\right)$ on the tongue surface, one of the chief causes of halitosis. The participants in this study who died of aspiration pneumonia demonstrated a greater level of $\mathrm{CH}_{3} \mathrm{SH}$ than participants in other groups. The number of Candida species on the lingual surface also tended to be higher in those subjects who died of aspiration pneumonia. High numbers of Candida species may have been associated with reduced host immunocompetence, which influenced resistance against virulent bacteria.

Of 23 patients evaluated after brain surgery [16], 5 developed postoperative aspiration pneumonia and were compared to the other 18 patients without postoperative lung complications with respect to their preoperative periodontal status and oral bacterial profile. Preoperative dental exams were performed to quantify the severity of the periodontal disease using a numeric scoring system. Periodontal conditions of the patients were categorized in five main diagnoses that were each given a numeric score. A "Disease Score" and "Severity Score" were also calculated based on the sum of the scores of coexisting periodontal diseases. Both the Disease Score and the Severity Score for periodontal disease were significantly greater in patients with postoperative pneumonia compared to the control group. The relative risk of developing postoperative pneumonia in patients with a high periodontal score was 3.5 times greater than in patients who had a low periodontal score, emphasizing the importance of preoperative oral health assessment to identify patients at risk, as this type of pneumonia could be prevented with proper oral care intervention.

\section{Association Between Oral Microbes and Respiratory Illnesses}

While studies examining the link between "bacterial pneumonia" and/or "aspiration pneumonia" and oral 
hygiene have yielded at least some neutral or negative results [13, 14], systematic reviews and recent studies strongly suggest a relationship. Of course, diagnosing pneumonia, in and of itself, can bring challenges, as definitions of diagnostic criteria vary from one hospital to another. Therefore, it is important to explore the more general association between oral microorganisms and respiratory illness as well. The study of the association between oral microorganisms and respiratory illnesses began as early as 1992 when Scannapieco et al. [17] conducted a study to assess the prevalence of oral colonization by respiratory pathogens in intensive care unit (ICU) patients, with specific attention to dental plaque and the oral mucosa. Quantitative cultures of dental plaque and buccal mucosa were obtained within $12 \mathrm{~h}$ of medical ICU admission and every third day thereafter until death or discharge from the ICU. Plaque scores demonstrated poor oral hygiene of the medical ICU patients compared to outpatients seen in a preventive dentistry clinic. Plaque and/or oral mucosa of 22 of 34 (65\%) medical ICU patients were colonized by respiratory pathogens compared to only 4 of $25(16 \%)$ preventive dentistry clinic patients. The potential respiratory pathogens cultured from medical ICU patients included methicillin-resistant Staphylococcus aureus, Pseudomonas aeruginosa, and ten types of Gramnegative bacilli. For the medical ICU group, contingency table analysis demonstrated a statistically significant ( $p W 0.05$ ) association between oral colonization by the respiratory pathogens and antibiotic therapy. The antibiotics used were ampicillin, clindimycin, ceftizoxime, and cefazolin. The relationship between the presence of dental plaque and respiratory pathogen colonization was examined within the medical ICU group. While dental plaque was present in large amounts in this group, the difference was not statistically significant $(p=0.185)$.

Similar results have been reported in elderly nursing home residents [18]. Plaque scores on teeth and dentures were significantly higher for the nursing home subjects than for the dental outpatient control (DOC) subjects. While no subjects in the DOC group were found to be colonized with respiratory pathogens, $14.3 \%$ (4/28) of the nursing home subjects were found to be colonized. Oral colonization with respiratory pathogens in the nursing home subjects was associated with the presence of chronic obstructive pulmonary disease (COPD) and higher plaque scores.

Data from the National Health and Nutrition Examination Survey I (NHANES I) were analyzed in a 1998 study by Scannapieco et al. [19]. This database contains information on the general health status of 23,808 individuals. Of these, 386 individuals reported a suspected respiratory condition that was further assessed by a physician. Subjects with confirmed chronic respiratory disease (chronic bronchitis or emphysema) or an acute respiratory disease (influenza, pneumonia, acute bronchitis) were compared to those not having a respiratory disease. The Oral Hygiene Index $(\mathrm{OHI})$ was utilized to assign a score to represent the patient's oral hygiene status, with a score of zero indicating good oral hygiene and a high score corresponding to poor oral hygiene. Results showed that subjects with a median OHI value were 1.3 times more likely to have a chronic respiratory disease than those with an OHI of zero. Subjects with the maximum $\mathrm{OHI}$ value were 4.5 times more likely to have a chronic respiratory disease.

In light of such findings, Scannapieco [20] proposed the following mechanisms for oral bacteria and respiratory infection: (1) aspiration of oral pathogens (e.g., Porphyromonas gingivalis and Actinobacillus actinomycetem comitans) into the lung causing infection; (2) periodontal disease-associated enzymes in saliva modifying mucosal surfaces to promote adhesion and colonization by respiratory pathogens, which are then aspirated into the lung; (3) periodontal disease-associated enzymes destroying salivary pellicles on pathogenic bacteria to hinder their clearance from the mucosal surface; and (4) cytokines originating from periodontal tissues which alter respiratory epithelium to promote infection by respiratory pathogens.

To further understand the relationship between underlying systemic diseases and the frequency of isolation of oral opportunistic pathogens, Senpuku et al. [21] conducted epidemiological studies of nursing home residents to determine the prevalence of bacteria and fungi causing aspiration pneumonia in association with oral biofilm bacteria. The influences of gender, age, denture-wearing status, number of teeth, and bedridden status in the patients were then analyzed. The isolation frequency rates of Candida albicans, Pseudomonadaceae, Staphylococcus spp., and some strains of Enterobacteriaceae in plaque samples, as well as $C$. albicans and Xanthomonas maltophilia in samples from the pharynx, were significantly higher ( $p W 0.05)$ in those requiring subacute care (mean age $=83.9$ years) than in those who did not require such care (mean age $=71.0$ years). In particular, the frequencies of Pseudomonas spp., C. albicans, and Serratia marcescens in plaque were significantly higher in those who were bedridden ( $p W 0.05$ ). The coexistence of Pseudomonas spp. and C. albicans in elderly persons with 10-19 teeth is a potential indicator for aspiration pneumonia and heart disease.

\section{Interventions to Improve Oral Care}

Oral care strategies for the average, healthy individual are well known: brush twice per day, floss once per day, and see the dentist every 6 months for a check-up. However, 
for medically compromised persons who are hospitalized or residing in nursing homes, these basics are often set aside and oral care becomes much more complex, especially when individuals cannot care for themselves. The question then becomes: What specific strategies can be used to achieve optimum oral health, particularly to avoid an increase in the incidence of aspiration pneumonia? The literature provides a number of references on the use of oral rinses and various methods of oral cleansing.

Literature reviews conducted in 2003 [12], 2006 [9], and 2008 [22] reported that improved oral hygiene and frequent professional oral health care reduces the progression or occurrence of respiratory diseases and the overall incidence of aspiration pneumonia by an average of $40 \%$ among high-risk elderly adults living in nursing homes in intensive care units (Level 1, grade A recommendation), though interventions included mechanical plaque removal (i.e., tooth brushing, swabbing), topical chemical disinfection, and/or use of antibiotics. Available results from randomized controlled trials linking oral hygiene status to pneumonia and respiratory tract infections in elderly people offer strong evidence that providing mechanical oral hygiene may prevent one in ten cases of death from pneumonia in dependent elderly people, and they indicate a largely similar effect on the prevention of pneumonia [22]. Breaking down the research into those investigating chemical versus mechanical intervention, therefore, seems warranted.

\section{Chemical Means of Intervention}

In the mid-1970s, $0.12 \%$ chlorhexidine gluconate (CHX) under the brand name of Peridex ${ }^{\circledR}$ was approved by the U.S. Food and Drug Administration and introduced for use by individuals undergoing periodontal treatment and dental implant surgery, as a presurgical and general antiseptic hand scrub [23, 24], umbilical cord cleanser, and for treating burns [25], cuts, and even acne. CHX is a broadspectrum antiseptic rinse that reduces both Gram-positive and Gram-negative bacteria. What is uniquely important about chlorhexidine is its substantivity, i.e., its ability to remain chemically active on tissue for up to $6 \mathrm{~h}$ [26]. Periodontists and oral surgeons prescribed the antimicrobial mouth rinse to decrease the oral bacterial burden for improved postsurgical healing and for long-term maintenance in some cases, such as dental implants. Additional uses of CHX in the oral cavity have included treatment of aphthous and herpetic ulcers [27], as an additive in dressings used for third molar extraction sites [28], as an irrigation for dry socket sites [29], and to assist in the management of oral conditions related to leukemia [30] and cancer patients receiving radiation therapy in the head and neck region.
In 1996, DeRiso et al. [31] conducted a study using $0.12 \% \mathrm{CHX}$ rinses for pre- and postoperative heart surgery patients. A 0.5-fluid-ounce oropharyngeal rinse was provided to the CHX group two times per day for 30-s rinses (no ingestion). If unable to rinse, the $\mathrm{CHX}$ was rigorously applied to the patient's buccal, pharyngeal, gingival, tongue, and tooth surfaces by a staff member. In the $\mathrm{CHX}$ group, results demonstrated a decrease in nosocomial infections by $65 \%$ and Gram-negative organisms involved in nosocomial infections by $59 \%$. CHX subjects also demonstrated a decrease in total respiratory tract infections by $69 \%$ and Gram-negative organisms associated with respiratory tract infections by $67 \%$. The use of nonprophylactic IV antibiotics decreased by $43 \%$ and there was a reduction in the mortality rate as well. There was no change in bacterial antibiotic resistance patterns for either subject group.

In a 2000 study [32], the European $0.2 \%$ chlorhexidine gluconate was applied in gel form to dentate patients in the intensive care unit who were mechanically ventilated. After mouth rinsing and oropharyngeal suctioning, the nurse applied the gel to tooth and gingival surfaces three times a day. The gel was left in place and the patient was instructed not to rinse, eat, or drink. Even without the mechanical elimination of plaque by tooth brushing, plaque scores progressively decreased, indicating a positive result for decreased plaque growth with the use of chlorhexidine gluconate. Because ICU patients are often immunologically compromised, the mechanical action of the toothbrush may place the patient at risk for bacteremia, which is sound reasoning for the use of $\mathrm{CHX}$ as the major source of oral decontamination. In this study, there was a decreased rate of ICU-related nosocomial infections as well as a decrease in ventilator-associated pneumonia.

ICU patients on mechanical ventilation were examined in a study [33] to determine the effect of decreased oropharyngeal colonization on the incidence of ventilatorassociated pneumonia (VAP). A study group was treated prophylactically with a topical antimicrobial mixture of $2 \%$ gentamycin, $2 \%$ colistin, and $2 \%$ vancomycin in an Orabase $^{\circledR}$ suspension. Orabase ${ }^{\circledR}$ without antibiotics was applied as a placebo to two control groups. The mixture was applied by gloved finger to the buccal cavities and oropharynx every $6 \mathrm{~h}$ beginning within $24 \mathrm{~h}$ of intubation. The study continued until extubation or death, and normal oral hygiene procedures were provided to all patients. In the study group, topical application of the Orabase ${ }^{\circledR}$ antibiotic mixture reduced the microbial colonization in the oropharynx by $75 \%$ and in the trachea by $52 \%$. Without affecting the gastrointestinal colonization, treatment prevented acquired oropharyngeal colonization by $10 \%$. Incidences of VAP were $10 \%$ in the study group, $31 \%$ in control group 1, and $23 \%$ in control group 2 . 
A more recent randomized controlled trial [26] that included 207 mechanically ventilated patients looked at the effectiveness of oral decontamination with $2 \%$ chlorhexidine (CHX) solution for the prevention of VAP. Patients in the chlorhexidine group received oral care four times per day that involved brushing the teeth, suctioning any oral secretions, and rubbing the oral mucosa with $15 \mathrm{ml}$ of a $2 \%$ chlorhexidine solution. Patients in a normal saline group received the same oral care regimen except that their procedures used normal saline solution instead of chlorhexidine solution. The incidence of VAP was $4.9 \%$ in the CHX group and $11.4 \%$ in the normal saline group. The mean number of cases of VAP was 7 episodes per 1,000 ventilator days in the CHX group and 21 episodes per 1,000 ventilator days in the normal saline group. In all patients, VAP was caused by Gram-negative bacilli with oropharyngeal colonization which was shown to be lower in the CHX group than in the normal saline group. The overall mortality rate for the patients in the CHX group was $32.3 \%$ compared with $35.2 \%$ for the normal saline group. Although oral decontamination with CHX reduced the risk of VAP in patients who received mechanical ventilation, no significant differences were noted regarding the duration of mechanical ventilation, length of ICU stay, or mortality rate. Nevertheless, oral decontamination with $\mathrm{CHX}$ for the prevention of VAP is considered a cost-effective strategy as the cost of the solution is far less than the cost of antibiotic therapy to treat an episode of VAP.

The effectiveness of a $0.12 \%$ chlorhexidine gluconate rinse versus Listerine ${ }^{\circledR}$ rinse was reported in 2007 [32]. In a group treated with $\mathrm{CHX}$, patients undergoing open heart surgery showed a $52 \%$ reduction in the rate of nosocomial pneumonia versus the Listerine ${ }^{\circledR}$ group. In patients who were intubated for over $24 \mathrm{~h}$, the rate of nosocomial pneumonia was $72 \%$ lower in the CHX group versus the Listerine ${ }^{\circledR}$ group. These results demonstrated a lower rate of nosocomial pneumonia for patients treated with chlorhexidine gluconate versus those treated with Listerine ${ }^{\circledR}$ rinse.

Only one study, to our knowledge, has reported negative findings with CHX [34]. In that study, $0.2 \%$ CHX gel was applied three times per day and did not reduce the incidence of VAP. However, inclusion criteria allowed patients with pre-existing infections to be enrolled. Sixty-eight percent of participants entered the study with exacerbated chronic bronchitis in COPD and/or community-acquired pneumonia.

Even though the majority of studies indicate positive results with the use of antimicrobial oral rinses for the reduction of aspiration pneumonia, the question remains as to if, when, how, and for whom a rinsing protocol should be implemented.

\section{Mechanical Means of Intervention}

The first mechanical line of defense is usually the toothbrush with the occasional addition of dental floss. Unfortunately, for persons who are ill, debilitated, and/or cognitively impaired, medical needs and other personal care needs outweigh oral care needs and even basic tooth brushing is forgotten or set aside. However, if improved oral care can improve or sustain a person's medical condition, specifically avoid aspiration pneumonia, it bears investigation.

Dentate and edentate subjects [35] were assigned to an oral care group or a no oral care group. After each meal, in the oral care group, nurses or caregivers cleaned the patients' teeth, palatal and mandibular mucosa, and tongue dorsum for $5 \mathrm{~min}$ by toothbrush. For patients with dentures or partials, the prostheses were cleaned with a denture brush after each meal and once per week with denture cleanser. For those patients unable to tolerate tooth brushing, the oropharynx was swabbed with $1 \%$ povidone iodine. Plaque and calculus removal was performed by dentists/dental hygienists once per week. At follow-up, pneumonia, febrile days, and death from aspiration pneumonia decreased in patients who received oral care. Interestingly, activities of daily living (ADLs) and cognitive functions also seemed to improve with oral care (see Table 3 for data comparisons between the oral care versus no oral care groups in dentate and edentate patients).

Professional oral care (POC) by dental professionals has been shown effective in reducing oral pathogens partly

Table 3 Comparisons between oral care versus no oral care in dentate and edentate patients

\begin{tabular}{lllllc}
\hline Patients & Group & No. of patients & $\begin{array}{l}\text { No. of patients } \\
\text { with fever }\end{array}$ & $\begin{array}{l}\text { No. of patients } \\
\text { with aspiration pneumonia }\end{array}$ & $\begin{array}{l}\text { No. of patients who died } \\
\text { from aspiration pneumonia }\end{array}$ \\
\hline Dentate & Oral care & 109 & $13(11 \%)$ & $12(9 \%)$ & $8(6 \%)$ \\
& No oral care & 99 & $26(26 \%)$ & $19(21 \%)$ & $20(20 \%)$ \\
Edentate & Oral care & 75 & $14(18 \%)$ & $9(9 \%)$ & $6(7 \%)$ \\
& No oral care & 83 & $28(34 \%)$ & $15(20 \%)$ & $10(13 \%)$ \\
\hline
\end{tabular}

From [35] 
responsible for aspiration pneumonia in medically compromised patients. To further define POC and clarify the optimum frequency with which it should be delivered, Ueda et al. [36] conducted a study of 105 nursing home patients. Of these patients, 55 were positive for oral Candida, which can be related to a number of possible factors, including poor oral hygiene, systemic malnutrition as opportunistic infection, or a fall in host resistance. POC was provided by dentists or dental hygienists via the use of an interdental brush, an "engine" brush (mechanical), and a scaler for calculus deposits. Sponge brushes were used to cleanse the tongue, palate, lips, and oral mucosa. For dentures and partials, surface debris was removed with a denture brush. Toothpastes and rinses were not used. The patients were divided into five groups and POC was provided at 1-, 2-, 3-, 4-, and 6-week intervals, respectively. Oral hygiene improvement rates decreased as POC intervals increased. Nursing homes generally do not have existing dental services (dentists and/or dental hygienists) to provide examinations or administer care. The researchers divided the results into three categories as follows: (1) short-term care, which consisted of POC provided once per week for 12 consecutive weeks, resulted in an overall improvement in the oral condition and eradication of Candida; (2) medium-term care, which consisted of POC provided once every 2 weeks for 20 weeks, resulted in overall improvement in oral condition and was considered to be the optimum interval for maintenance; (3) long-term care, which consisted of POC provided once every 34 weeks and was beneficial only if the patient's Functional Independence Measure (FIM) was over 3, indicating that the patient could take responsibility for his/her own effective oral care.

Another study [37] sought to determine whether improved oral care had any effect on the cough reflex and, ultimately, on aspiration pneumonia. Fifty-nine elderly nursing home patients were enrolled: 30 in the intervention group and 29 in the control group. Subjects in the intervention group were provided oral care (mechanical cleaning of teeth and gingiva) by caregivers after each meal for 1 month. The control group subjects performed their own oral care for the same period of time. Citric acid was used to test the cough reflex sensitivity of all subjects for baseline measurements and again at the end of the 30 days. End results for the intervention group showed higher cough reflex sensitivity than at baseline as well as higher sensitivity levels than the control group. This indicates that if aspiration pneumonia and cough are related, then improved oral care can increase cough reflex sensitivity, which in turn may decrease the potential for aspiration pneumonia.

Adachi et al. [38] conducted a study of elderly patients from two nursing homes. Professional oral hygiene care (POHC) was provided once per week by dental hygienists and included mechanical cleaning with electric toothbrushes with an automatic water supply, an interdental brush, and a sponge brush used on the teeth, buccal mucosa, tongue, and dentures. Nurses took the body temperatures of the subjects daily at 7:00 a.m. and 3:00 p.m. Six-month results indicated that POHC reduced the prevalence of fevers and lowered the prevalence of fatal pneumonia in the test group more so than in the control group. Influenza rate was also reduced, as were the numbers of anaerobic bacteria and the enzymatic activities in saliva, which can inhibit absorption of the cold virus into airway membranes.

The Toothette ${ }^{\circledR}$, a soft sponge on a swab-like stick, is frequently used by nurses and Certified Nurse Assistants (CNAs) for oral care in hospitals and nursing homes. Unfortunately, the Toothette ${ }^{\circledR}$ does not remove plaque as effectively as tooth brushing, and since tooth brushing skills are generally not taught to nurses and support staff, the proliferation of bacteria can occur. Fields [39] compared the rates of VAP in patients whose teeth were brushed three times a day (every $8 \mathrm{~h}$ ) with those of patients who received daily tooth brushing and oral care with Toothette ${ }^{\circledR}$. Patients in the control group received "usual care," which could include daily tooth brushing along with the use of the Toothette ${ }^{\circledR}$ as needed. For the intervention group, nurses were instructed on the importance of oral care and told to brush the patient's teeth, tongue, and hard palate with a toothbrush and toothpaste for at least $1 \mathrm{~min}$ at three specified times during the day. They were then to use the Toothette ${ }^{\circledR}$ to swab the patient's teeth, tongue, and hard palate for at least $1 \mathrm{~min}$. The VAP rate for the intervention group dropped to $0 \%$ per 1,000 ventilator days and was sustained for 6 months, demonstrating the efficacy of tooth brushing as a means to remove plaque-harboring bacteria, thus preventing VAP.

One study - conducted in 2009 [40] to determine the effectiveness of adding the use of an electric toothbrush to standard oral care with $0.12 \%$ chlorhexidine digluconate for the prevention of VAP-yielded negative results for mechanical intervention. Two groups of comparable patients $(n=147)$ were studied and the findings demonstrated that the addition of electric tooth brushing to standard oral care with $0.12 \%$ chlorhexidine digluconate was not effective for the prevention of VAP in that the groups did not differ significantly in mortality, antibiotic-free days, duration of mechanical ventilation, or hospital ICU length of stay $(\mathrm{CI}=0.41-1.73)$.

Given the overall conclusions of the reviewed literature that supports improved oral care and its relationship to the decreased incidence of respiratory pathogens, the next course of inquiry regarding nursing home patients is to determine who is responsible for daily oral care and how will it be implemented. 


\section{Oral Care Training for Nursing Staff}

Regarding the provision of oral care to nursing home patients, education of nurses and particularly CNAs may be part of the problem. In 1995, Hardy et al. [41] conducted a survey of 14 nursing homes in Virginia to determine the role of nurses and nurses' aides in the provision of oral hygiene care to the residents. Nurses' aides typically provide the oral health services and the majority reported that patient uncooperativeness was a major factor $(88 \%)$ in whether oral services were provided. In rating their knowledge of mouth rinses, denture cleaners, tooth brushing, fluoride rinses, and oral exams, 90-99\% rated their knowledge as adequate or excellent, with knowledge of saliva substitutes and flossing as poor.

Peltola et al. [42] examined a more general nursing home population in the U.S. by interviewing residents about current dental problems and dental services provided to them. Of the 412 residents interviewed, less than half were ambulant, over $70 \%$ had not seen a dentist for over 5 years, and $22 \%$ reported a current dental problem. Eighty-two percent of denture wearers were unable to clean their dentures, yet the staff cleaned dentures for only $64 \%$. Ninety-five percent of dentures were unhygienic and $33 \%$ were affected by stomatitis. Among dentate participants, $75 \%$ were unable to clean their teeth, yet none received regular assistance. Two-thirds of all tooth surfaces were covered in plaque, and periodontis was moderate to severe in most. Calculus and root caries were present in 82 and $63 \%$, respectively.

Binkley et al. [43] conducted a survey of nurses' attitudes and beliefs regarding oral care in 102 intensive care units in the U.S. Oral care was rated as a high priority, especially for mechanically ventilated patients. Of the nurses surveyed, $63 \%$ found the task of oral care to be difficult and $43 \%$ found it to be unpleasant. The nurses ranked high in their knowledge of the importance of oral care in relation to potential aspiration of pathogens into the lungs. In terms of oral care training, $88 \%$ stated adequate training, $67 \%$ reported that training was provided in nursing school, and $48 \%$ indicated that they were self-taught. The types and frequencies of oral care provided were variable, even within each institution. Foam swabs, mouthwashes, and moisture agents were the most commonly used materials, with manual toothbrushes and toothpaste used once per day by $40 \%$ of the respondents. Eighty-one percent of the nurses responded that they had adequate time to provide oral care to their patients once per day and that oral hygiene supplies were readily available; however, $46 \%$ indicated that better supplies or equipment were needed.

In a subsequent study [44], edentate patients in longterm care showed moderate $(44 \%)$ to poor $(37 \%)$ dental hygiene, with denture replacements needed in about $25 \%$ of them; and stomatitis and angular cheilitis were noted as 25 and $28 \%$, respectively. For dentate patients, $37 \%$ needed restorative work, $51 \%$ needed periodontal care, and $42 \%$ needed extractions.

The problem of resistant behavior was confirmed in an observational study conducted by Coleman [45] wherein mostly female patients (age range $=66-96$ years) with dementia and poor oral hygiene were observed. Teeth were brushed and mouth rinsed with water $16 \%$ of the time; only one resident had her tongue brushed. Standards were never met with regard to 2-min brushing time, flossing, oral assessment, rinsing with mouthwash, or wearing clean gloves. Oral care supplies were not available most of the time. Most of the residents were resistant to efforts to provide oral care and disruptive behaviors were common.

Thean et al. [46] completed a pilot self-administered questionnaire for 53 nursing staff in a nursing home to assess their knowledge of dental decay, periodontal disease, and the care of dentures. He found a positive attitude by $99 \%$ of the respondents regarding the importance of providing oral care; however, only a third of the staff had received training in oral health and the management of oral care for the nursing home residents. While the staff had a good understanding (88\%) of the role of plaque in the development of periodontal disease, only $45 \%$ understood the relationship of sugary foods and drinks to the formation of dental caries. Most of the staff (96\%) agreed that dentures should be cleaned, but only half understood that broken dentures could/should be repaired.

Chiba et al. [47] conducted a survey of 102 caregiver managers to investigate their knowledge, practice, and educational background regarding oral health. The data collected were also used to analyze the relationship between factors of oral health education and length of career. Results indicated that the length of career for home-care staff was approximately 3.5 years and that for caregiver managers was 1.6 years. Ninety percent recognized the importance of oral care and were interested in oral care, although $32.4 \%$ hesitated to provide oral care. The response rate of those subjects who knew that soft debris was bacterial plaque was under $50 \%$. Generally speaking, the lack of knowledge and skill of professional care may be related to the length of the career of care professionals.

\section{Today and Tomorrow}

Dental care to promote prevention has evolved in the past 20 years to include numerous products for improved oral 
hygiene and preventive approaches such as fluoridated water supplies and in-office procedures such as pit and fissure sealants. With these improvements, people are able to keep their natural dentition longer, maybe for life. In the future, edentulousness may someday be a rare phenomenon with more elderly dentate people. While these individuals may have more teeth, they may very well have impaired ability to perform oral hygiene and thus more cases of periodontal disease. It is also this population who will be at high risk for pulmonary infections. To assure that improved knowledge and methods to save people's natural teeth are not taking the elderly from one problem to another, emphasis on the importance of good oral hygiene and the prevention of periodontal disease is crucial [48].

Unbeknownst to many, Medicare provides minimal coverage for dental care. This needs to be changed, especially in light of the current research on the connection between periodontal disease and systemic diseases, as well as the issues raised in this review. Lobbyists for the American Speech-Language and Hearing Association, the American Dental Hygienists' Association, and the American Dental Association should work together to advocate for reforms to Medicare coverage to include the provision of dental hygiene and dental services to the elderly. In addition, access to dental hygiene services in the nursing home setting is poor given the supervision restrictions placed on dental hygienists, an area where legislation at the state level must be addressed and changed.

As research continues to advance the evidence base on oral care for the elderly, education at every level is equally important to increase awareness, promote improved care, advocate for increased access to care, and enrich the quality of life for the elderly. This education should begin in training programs for nurses, dental hygienists, certified nurse assistants, and public health professionals and continue via professional organizations to public health agencies and branches of government responsible for implementing policy changes.

Changes in health policies have stressed the importance of evidence-based clinical practice and the need to evaluate outcomes that are significant to patients. These changes are particularly relevant for the underserved elderly, especially those residing in long-term-care facilities. Evidence clearly demonstrates a connection between oral microorganisms, dental biofilms, respiratory pathogens, and aspiration pneumonia in this population. Further research is certainly indicated.

Raghavendran et al. [49] suggested a need for the following research areas to be addressed: (1) define what is minimally required to reduce the risk of aspiration pneumonia for all patients, including denture wearers, those who are tube-fed, nonambulatory, or suffering with a dementing illness who may be resistant to oral care; (2) examine gingival inflammation and the resulting release of cytokines and proteases into oral secretions which may increase the risk for aspiration pneumonia; (3) determine which methods are most effective and practical for mechanical removal of dental plaque and biofilms: swabs, manual toothbrushes, or electric toothbrushes, as well as the role of flossing. Frequency and duration of care need to be further investigated as well as the training and credentials of those providing the care.

To enhance clinical practice based on evidence thus far, Raghavendran et al. [49] suggest combining mechanical oral cleansing with antimicrobial rinsing, as rinsing alone is insufficient to remove biofilms laden with harmful microorganisms. Chlorhexidine gluconate antimicrobial mouth rinse has been shown to be effective for the reduction of oropharyngeal microbes; however, it contains alcohol, a known drying agent for mucosal tissues. Many medications cause xerostomia, so an alcoholfree rinse such as Crest ProHealth ${ }^{\circledR}$ may be indicated for those patients. Oral moisturizers and saliva substitutes such as Biotene ${ }^{\circledR}$ are viable adjuncts for patients with xerostomia.

In the nursing home setting, education, organization, and time management are key factors. The authors of this article have proposed a method to improve the provision of oral care services to the nursing home elderly as well as provide documentation of problems noted that need the attention of a dental care specialist. These procedures can be found in the Appendix.

The profession of dental hygiene has recently issued publications on the link between oral health and respiratory diseases to heighten the awareness of dental hygienists. These articles point out the various oral care interventions discussed in this review as well as implications for the role of the dental hygiene professional to work with caregivers, nurses, and other health-care professionals to increase the quality of oral health and general health $[48,50,51]$.

The need for a multidisciplinary approach to address this problem is evident. Nurses, CNAs, speech-language pathologists, and dental hygienists all have a knowledge base and clinical experience that when combined, can positively impact the lives and well-being of the elderly. As Coleman [45] so eloquently pointed out, "CNA's need strategies that will allow them to deliver oral care effectively and humanely for residents who resist their efforts." The increase of knowledge base and the provision of appropriate training will empower health-care professionals to improve the oral health of their patients and, in doing so, decrease the risk of aspiration pneumonia and other respiratory illnesses, and potentially save lives. 


\section{Appendix}

Forms Used to Document Oral Care for the Nursing

Home Patient

Nursing Home Staff Oral Care Instructions

\begin{tabular}{|c|c|}
\hline CNA'S & NURSE SUPERVISORS \\
\hline $\begin{array}{l}\text { * Attend Inservice to be provided by speech pathologist/dental } \\
\text { hygienist. } \\
* \text { Instruct patients and caregivers - enlist their assistance. }\end{array}$ & $\begin{array}{l}\text { * Attend Inservice to be provided by speech } \\
\text { pathologist/dental hygienist. } \\
* \text { Make sure staff attends. } \\
* \text { With new staff, schedule another inservice } \\
\text { or provide information to them directly. }\end{array}$ \\
\hline $\begin{array}{l}\text { * Be knowledgeable about the inventory system. } \\
\text { * When running low, ask for order to be placed. } \\
\text { * Supplies needed in inventory: } \\
\text { - } \quad \text { Gloves/masks } \\
\text { - } \quad \text { Electric toothbrushes }\left(\text { Crest Spin Brushes }{ }^{\circledR} \text { ) }\right. \\
\text { - Denture brushes and containers } \\
\text { - } \quad \text { Denture cleansers } \\
\text { - } \quad \text { Disponge swabs } \\
\text { - Peridex }{ }^{\circledR} \text { (chlorhexidine gluconate) } \\
\text { - Crest Pro-Health }{ }^{\circledR} \\
\text { - } \quad \text { Saliva substitute. }\end{array}$ & $\begin{array}{l}\text { * Develop an inventory system for oral care } \\
\text { supplies. } \\
\text { * Make sure supplies are ordered in a timely } \\
\text { fashion. } \\
\text { * Make sure supplies are easily accessible to } \\
\text { staff. }\end{array}$ \\
\hline $\begin{array}{l}\text { Provide oral care for pts } 2 \mathrm{X} / \text { day: } \\
\text { - } \quad \text { morning after breakfast } \\
\text { - } \quad \text { at night before bed. }\end{array}$ & $\begin{array}{l}\text { Develop a timeline for staff to follow for oral care } 2 \\
\text { times/day. }\end{array}$ \\
\hline $\begin{array}{l}\text { Oral Care Regimen for dentate patients: } \\
\text { Mechanical: } \\
\text { - } \quad \text { Brushing - use electric toothbrush for teeth and tongue } \\
\text { - } \quad \text { Sponge swab for mucosa (cheeks, lips) } \\
\text { - Flossing - use flossers once/day. } \\
\text { Rinsing. } \\
\text { - Peridex - for patients with inflamed gums } \\
\text { - Crest ProHealth - (alcohol-free) for patients with dry } \\
\text { - } \text { mouth }\end{array}$ & $\begin{array}{l}\text { * Divide patients and assign (number of patients) } \\
\text { patients per staff member. } \\
\text { * Have assigned staff check off tasks as } \\
\text { completed during the day (see checklist). } \\
\text { * Copy checklists for each patient and } \\
\text { provide to staff daily. }\end{array}$ \\
\hline $\begin{array}{l}\text { Care for Dentures/Partials: } \\
\text { Mechanical: } \\
\text { Use denture brush to clean prostheses. Place in ultrasonic cleaner } \\
\text { with solution for } 10 \text { minutes. } \\
\text { Rinsing: } \\
\text { Have patients rinse (without prostheses) as indicated above. }\end{array}$ & $\begin{array}{l}\text { * Obtain ultrasonic cleaner and solution for the purpose } \\
\text { of cleaning dental prostheses. }\end{array}$ \\
\hline $\begin{array}{l}\text { Oral assessment - weekly } \\
\text { * Take a good look in the mouth, palpate lips and cheeks with } \\
\text { fingers to assess for the following: } \\
\text { - Holes in teeth (cavities) } \\
\text { - Red, swollen gums, bad breath (gum disease, denture } \\
\text { stomatitis) } \\
\text { - Redness, cracking at corners of mouth (especially denture } \\
\text { - } \text { patients) } \\
\text { Sores, lumps. }\end{array}$ & $\begin{array}{l}\text { * Develop a timeline (a specific day) for staff } \\
\text { to follow for oral assessment once/week. } \\
* \text { Keep copies of referral forms available for } \\
\text { staff. }\end{array}$ \\
\hline $\begin{array}{l}\text { If you see suspicious area, complete referral form and submit to } \\
\text { nurse supervisor. }\end{array}$ & $\begin{array}{l}\text { * Develop relationship with dentist/dental hygienist who } \\
\text { can visit NH to examine/treat patients regularly. }\end{array}$ \\
\hline
\end{tabular}


Patient Name:

Daily Care

\begin{tabular}{|l|l|l|}
\hline AM & PM & \multicolumn{1}{|c|}{ TASK } \\
\hline & & $\begin{array}{l}\text { For dentate patients: } \\
\text { Brush - use electric toothbrush for teeth and tongue }\end{array}$ \\
\hline & --- & Floss - use flossers once/day \\
\hline & & $\begin{array}{l}\text { Peridex - for patients with inflamed gums } \\
\text { OR } \\
\text { Crest ProHealth - (alcohol-free) for patients with dry mouth }\end{array}$ \\
\hline & & $\begin{array}{l}\text { Saliva substitute - for patients with dry mouth } \\
\text { Use denture brush, ultrasonic cleaner to clean prostheses }\end{array}$ \\
\hline & & \begin{tabular}{l} 
Have patients rinse (without prostheses) as indicated above \\
\hline
\end{tabular} \\
\hline
\end{tabular}

\begin{tabular}{|c|l|} 
Weekly Assessment \\
\hline Monday & $\begin{array}{l}\text { Take a good look in the mouth! } \\
\text { Palpate (feel) lips and cheeks with fingers! }\end{array}$ \\
\hline--- & Holes in teeth (cavities) \\
\hline & Red, swollen gums, bad breath (gum disease, denture stomatitis) \\
\hline & Redness, cracking at corners of mouth (especially for denture patients) \\
\hline & $\begin{array}{l}\text { Note suspicious items on referral form and submit to nurse supervisor } \\
\text { Make note of supplies needed and request nurse supervisor to order }\end{array}$ \\
\hline
\end{tabular}

Written Comments:

(presence of fever, patient/caregiver complaints, resistive behaviors, compliance tips)

Staff Member:

\section{References}

1. Oh E, Weintraub N, Dhanani S. Can we prevent aspiration pneumonia in the nursing home? J Am Med Dir Assoc. 2004;5:174-9.

2. Wilkins EM. Clinical practice of the dental hygienist. 10th ed. Philadelphia: Lippincott Williams \& Wilkins; 2009.

3. Nield-Gehrig JS, Willmann DE. Foundations of periodontics for the dental hygienist. 2nd ed. Baltimore: Lippincott Williams \& Wilkins; 2008.

4. Socransky SS, Haffajee AD. The bacterial etiology of destructive periodontal disease: current concepts. J Periodontol. 1992;63(4 Suppl):322-31.

5. Socransky SS, Manganiello SD. The oral microbiota of man from birth to senility. J Periodontol. 1971;42(8):485-96.
6. Tanner AC, Paster BJ, Lu SC, Kanasi E, Kent R Jr, Van Dyke T, Sonis ST. Subgingival and tongue microbiota during early periodontitis. J Dent Res. 2006;85(4):318-23.

7. Haffajee AD, Cugini MA, Tanner A, Pollack RP, Smith C, Kent RL Jr, Socransky SS. Subgingival microbiota in healthy, wellmaintained elder and periodontitis subjects. J Clin Periodontol. 1998;25(5):346-53.

8. Cavallazzi R, Vasu TS, Marik PE. Aspiration pneumonitis and aspiration pneumonia. Dysphagia. 2009;18(1):25-33.

9. Azarpazhooh A, Leake JL. Systematic review of the association between respiratory diseases and oral health. J Periodontol. 2006;77(9):1465-82.

10. Langmore SE, Terpenning MS, Schork A, Yinmiao C. Predictors of aspiration pneumonia: how important is dysphagia? Dysphagia. 1998;13(2):69-81. 
11. Langmore SE, Skarupski KA, Park PS, Fries BE. Predictors of aspiration pneumonia in nursing home residents. Dysphagia. 2002;17(4):298-307.

12. Scannapieco FA, Bush RB, Paju S. Associations between periodontal disease and risk for nosocomial bacterial pneumonia and chronic obstructive pulmonary disease. A systematic review. Ann Periodontol. 2003;8(1):54-69.

13. Chabrand F, Allamand JM, Duroux P, Rabate B, Laudenbach P, Doyon D. Are orodental infectious foci responsible for bacterial pneumopathies? A statistical study. Rev Stomatol Chir Maxillofac. 1986;87:73-7.

14. Treloar DM, Stechmiller JK. Use of a clinical assessment tool for orally intubated patients. Am J Crit Care. 1995;4:355-60.

15. Awano S, Ansai T, Takata Y, Soh I, Akifusa S, Hamasaki T, Yoshida A, Sonoki K, Fujisawa K, Takehara T. Oral health and mortality risk from pneumonia in the elderly. J Dent Res. 2008;87(4):334-9.

16. Bagyi K, Haczku A, Marton I, Szabo J, Gaspar A, Andrasi M, Varga I, Toth J, Klekner A. Role of pathogenic oral flora in postoperative pneumonia following brain surgery. BMC Infect Dis. 2009;9:104.

17. Scannapieco FA, Stewart EM, Mylotte JM. Colonization of dental plaque by respiratory pathogens in medical intensive care patients. Crit Care Med. 1992;20(6):740-5.

18. Russell SL, Boylan RJ, Kaslick RS, Scannapieco FA, Katz RV. Respiratory pathogen colonization of the dental plaque of institutionalized elders. Spec Care Dentist. 1999;19(3):128-34.

19. Scannapieco FA, Papandonatos GD, Dunford RG. Associations between oral conditions and respiratory disease in a national sample survey population. Ann Periodontol. 1998;3(1):251-6.

20. Scannapieco FA. Role of oral bacteria in respiratory infection. J Periodontol. 1999;70(7):793-802.

21. Senpuku H, Sogame A, Inoshita E, Tsuha Y, Miyazaki H, Hanada $\mathrm{N}$. Systemic diseases in association with microbial species in oral biofilm from elderly requiring care. Gerontology. 2003;49(5): $301-9$.

22. Sjogren P, Nilsson E, Forsell M, Johansson O, Hoogstraate J. A systematic review of the preventive effect of oral hygiene on pneumonia and respiratory tract infection in elderly people in hospitals and nursing homes: effect estimates and methodological quality of randomized controlled trials. J Am Geriatr Soc. 2008;56(11):2124-30.

23. Furukawa K, Ogawa R, Norose Y, Tajiri T. A new surgical handwashing and hand antisepsis from scrubbing to rubbing. J Nippon Med Sch. 2004;71(3):190-7.

24. Weber DJ, Sickbert-Bennett E, Gergen MF, Rutala WA. Efficacy of selected hand hygiene agents used to remove Bacillus atrophaeus (a surrogate of Bacillus anthracis) from contaminated hands. JAMA. 2003;289(10):1274-7.

25. Fraser JF, Bodman J, Sturgess R, Faoagali J, Kimble RM. An in vitro study of the anti-microbial efficacy of a $1 \%$ silver sulphadiazine and $0.2 \%$ chlorhexidine digluconate cream, $1 \%$ silver sulphadiazine cream and a silver coated dressing. Burns. 2004;30(1):35-41.

26. Tantipong H, Morkchareonpong C, Jaiyindee S, Thamlikitkul V. Randomized controlled trial and meta-analysis of oral decontamination with $2 \%$ chlorhexidine solution for the prevention of ventilator-associated pneumonia. Infect Control Hosp Epidemiol. 2008;29(2):131-6.

27. Park JB, Park NH. Effect of chlorhexidine on the in vitro and in vivo herpes simplex virus infection. Oral Surg Oral Med Oral Pathol. 1989;67(2):149-53.

28. Fotos PG, Koorbusch GF, Sarasin DS, Kist RJ. Evaluation of intra-alveolar chlorhexidine dressings after removal of impacted mandibular third molars. Oral Surg Oral Med Oral Pathol. 1992;73(3):383-8.
29. Field EA, Nind D, Varga E, Martin MV. The effect of chlorhexidine irrigation on the incidence of dry socket: a pilot study. $\mathrm{Br}$ J Oral Maxillofac Surg. 1988;26(5):395-401.

30. Shepherd JP. The management of the oral complications of leukemia. Oral Surg Oral Med Oral Pathol. 1978;45(4):543-8.

31. DeRiso AJ, Ladowski JS, Dillon TA, Justice JW, Peterson AC. Chlorhexidine gluconate $0.12 \%$ oral rinse reduces the incidence of total nosocomial respiratory infection and nonprophylactic system antibiotic use in patients undergoing heart surgery. Chest. 1996;109(6):1556-61.

32. Fourrier F, Cau-Pottier E, Boutigny H, Roussel-Delvallez M, Jourdain M, Chopin C. Effects of dental plaque antiseptic decontamination on bacterial colonization and nosocomial infections in critically ill pts. Intensive Care Med. 2000;26(9):1239-47.

33. Bergmans DC, Bonten MJ, Gaillard CA, Paling JC, van der Geest S, van Tiel FH, Beysens AJ, de Leeuw PW, Stobberingh EE. Prevention of ventilator-associated pneumonia by oral decontamination: a prospective, randomized, double-blind, placebocontrolled study. Am J Respir Crit Care Med. 2001;164(3):382-8.

34. Fourrier F, Dubois D, Pronnier P, Herbecq P, Leroy O, Desmettre T, Pottier-Cau E, Boutigny H, Di Pompéo C, Durocher A, Roussel-Delvallez M, PIRAD Study Group. Effect of gingival and dental plaque antiseptic decontamination on nosocomial infections acquired in the intensive care unit: a double-blind placebo-controlled multicenter study. Crit Care Med. 2005;33:1728-35.

35. Yoneyama T, Yoshida M, Ohrui T, Mukaiyama H, Okamoto $H$, Hoshiba K, Ihara S, Yanagisawa S, Ariumi S, Morita T, Mizuno Y, Ohsawa T, Akagawa Y, Hashimoto K, Sasaki H, Oral Care Working Group. Oral care reduces pneumonia in older patients in nursing homes. J Am Geriatr Soc. 2002;50(3):430-3.

36. Ueda K, Toyosato A, Nomura S. A study on the effects of short-, medium-, and long-term professional oral care in elderly persons requiring long-term nursing care at a chronic or maintenance stage of illness. Gerodontology. 2003;20(1):50-6.

37. Watando A, Ebihara S, Ebihara T, Okazaki T, Takahashi H, Asada M, Sasaki H. Daily oral care and cough reflex sensitivity in elderly nursing home patients. Chest. 2004;126(4):1066-70.

38. Adachi M, Ishihara K, Abe S, Okuda K. Professional oral health care by dental hygienists reduced respiratory infections in elderly persons requiring nursing care. Int J Dent Hyg. 2007;5:69-74.

39. Fields LB. Oral care intervention to reduce incidence of ventilator-associated pneumonia in the neurologic intensive care unit. J Neurosci Nurs. 2008;40(5):291-8.

40. Pobo A, Losboa T, Rodriguez A, Sole R, Magret M, Trefler S, Gomez F, Rello J. A randomized trial of dental brushing for preventing ventilator-associated pneumonia. Chest. 2009;136(2): 433-9.

41. Hardy DL, Darby ML, Leinbach RM, Welliver MR. Self-report of oral health services provided by nurses' aides in nursing homes. J Dent Hyg. 1995;69(2):75-82.

42. Peltola P, Vehkalahti MM, Wuolijoki-Saaristo K. Oral health and treatment needs of the long-term hospitalized elderly. Gerodontology. 2004;21(2):93-9.

43. Binkley C, Furr LA, Carrico R, McCurren C. Survey of oral care practices in US intensive care units. Am J Infect Control. 2004;32(3):161-9.

44. Paju S, Scannapieco FA. Oral biofilms, periodontitis, and pulmonary medicine. Oral Dis. 2007;13:508-12.

45. Coleman P. Resistive behaviors of elderly nursing home residents during oral care. Geriatr Nurs. 2005;26(6):349-50.

46. Thean $\mathrm{H}$, Wong ML, Koh $\mathrm{H}$. The dental awareness of nursing home staff in Singapore - a pilot study. Gerodontology. 2007;24:58-63.

47. Chiba Y, Shimoyama K, Suzuki Y. Recognition and behavior of caregiver managers related to oral care in the community. Gerodontology. 2009;26:112-21. 
48. Agado B, Bowen DM. Does the link between COPD and periodontitis affect dental hygiene treatment? Access. 2009;23(4): $19-21$.

49. Raghavendran K, Mylotte JM, Scannapieco FA. Nursing homeassociated pneumonia, hospital-acquired pneumonia and ventilator-associated pneumonia: the contribution of dental biofilms and periodontal inflammation. Periodontol 2000. 2007;44:164-77.

50. Elliot-Smith S. Finding a collaborative link: treating periodontal and respiratory diseases. Access. 2009;23(4):16-8.
51. Gluch JI. Exploring the connection: the relationship between respiratory diseases and oral health. Dimens Dent Hyg. 2009; 7(10):54-7.

Cherin C. Pace MS, RDH, CCC-SLP

Gary H. McCullough PhD, CCC-SLP 